\title{
La poétique des Cinq Grandes Odes
}

\author{
Marie-Joséphine Whitaker
}

Une division importante, établie par Claudel lui-même au sein de son œuvre, met d'emblée en relief la nature propre des Odes, et leur assigne une place dans l'évolution de son génie (que le poète nomme modestement «talent ») :

L'élément subjectif dominait chez moi jusqu'en 1909 [...] À partir de 1910, la géologie de mon talent est changée [...] La composition domine l'inspiration (M.I. p. 251252)

Mais avant 1910, c'est-à-dire dans la phase où se situent les Odes, c'est manifestement le contraire qui est vrai : l'inspiration prime et gouverne tout, et répand partout ce « vin de gloire » (Odes, 264) qui enivre tout de suite le lecteur. Tout, dans les Odes - leur impétuosité sauvage, leur incohérence sublime, leur naturel intense et grandiose, tout y annonce la poésie même de l'inspiration. Nourri d'une même " veine vive " (Odes, 236) et né d'un même "impetus ", d'une même "poussée intérieure » (M.I. 251) le poème n'a pu que commencer et finir avec elle :

J'ai agi - confie Claudel à Jean Amrouche à propos de la première Ode - mon Dieu presque toujours par inspiration, sans que je puisse me rendre un compte exact de mes idées. C'est comme ça que je m'exprimais à ce moment-là. (Ibid., 184-185)

Et la même obéissance à l'inspiration détermine la fin du poème : 
J'ai fini mes Cinq Grandes Odes [...] J'en avais commencé une sixième, mais j'ai senti qu'il fallait s'arrêter. Je ne force jamais l'inspiration, (écrit Claudel à Gabriel Frizeau en 1908) ${ }^{1}$.

Fidélité à l'inspiration qui n'est qu'une autre forme de cette soumission à la vie qui fait loi et norme dans l'univers claudélien. Poésie, vie, source; pourrait-on mieux exprimer cette équation que ne le fait l'image suivante?

Que le langage nouveau, comme un lac plein de sources Déborde par toutes ses coupures! (226)

Toute la « doctrine " claudélienne de l'inspiration-expiration, si obscure lorsqu'on veut lui donner un sens trop précis, si claire dès qu'on y reconnaît une métaphore multiple rattachant l'action d'aspirer l'air par où débute toute existence aux origines de l'art - Muses « respiratrices » et inspirées, tentatives à demi convaincantes de fonder une prosodie sur la respiration, identification du souffle et de l'Esprit (O.P. 189) - tout ceci n'a qu'un but : unifier l'esthétique claudélienne sous un seul principe de vie. Car toute démarche du poète débouche sur un certain «vie-isme » ou vitalisme ${ }^{2}$ : l'inspiration, dans ce "système ", qu'elle se nomme " grâce " (V. Pr. 54) ou vie n'est qu'une autre fréquence du courant vital, s'intensifiant en poésie.

L'inspiration poétique [...] n'est que l'accentuation d'un phénomène plus général [...] La poésie est partout. Il n'y a pas de phénomène à des degrés divers d'intensité plus qénéral et plus étendu. (Pr. p. 54-55; 1393)

La poétique claudélienne accorde immédiatement ses pleins droits à l'inspiration. Une image forte (encore qu'empreinte d'humour), celle de la tête du roi Penthée, décapité pour avoir résisté à l'ivresse, le rappelle (269). Le plus souvent, la question ne se pose même pas : "instrument où l'on souffle » (225), jouet de 


\section{La Poétique des Cinq Grandes Odes}

l'inspiration, ou, pour employer ses propres symboles, cavalier impuissant à maîtriser son coursier, désarçonné, dépassé. par cette force plus prompte que toute faculté humaine, le poète est contraint à

courir comme (il peut) la main sur l'échine de ce quadrupède ailé qui (l') entraîne dans sa course cassée qui est à moitié aile et bond. (266)

Attitude grotesque et inconvenante! Mais qui ne voudrait d'un tel ridicule, qui provient de la disproportion entre l'humain et le divin, l'état de la créature et le don qui lui est fait? Tout est là : là est la force et la faiblesse des Odes, l'origine de leur splendeur comme de leurs inégalités. Le poète classique, qui, lui, est à pouvoir égal avec son inspiration (mince « jet d'une étroite cuvette » dira. Claudel, Pr. 31) n'a pas à subir d'humiliations mais n'est pas non plus gâté par les Muses. Le rang que la critique accordera aux Odes, inférieur ou supérieur à une poésie de bonne tenue, dépendra en définitive de ce qu'elle admettra ou non le fait de l'inspiration

Quant à Claudel lui-même, il n'y a aucun doute : il a choisi, dans les Odes, le ridicule de la défaite formelle plutôt que celui, moins voyant mais plus grave, de la médiocrité poétique. C'est ce que nous apprennent la mimique comme les paroles de la Muse dans la quatrième Ode : l'absurdité est encore plus grande à devancer la vie, précéder l'inspiration au lieu de la suivre :

Debout! marche devant moi, je le veux [lui ordonnet-elle] / Afin que je regarde et rie, et que j'imite, moi, la déesse, ton avancement mutilé! (272)

Claudel qui connaît si bien la condition de son art : l'inspiration ou rien, ne s'y est pas toujours tenu. En poésie, ce qui est né de l'occasion est toujours imparfait, notait Baudelaire (O.C. 714) et l'CEuvre poétique, de par la force des choses, comportera un certain nombre de vers de circonstance. 


\section{Marie-Joséphine Whitaker}

$$
\begin{aligned}
& * \\
& * * *
\end{aligned}
$$

Mais dans les Odes « pas à craindre d'être laissé au sec » (272) : c'est à peine si le poète peut nous parler d'autre chose que de cette puissance qui le soumet et le harcèle, tantôt Muse, tantôt mer (237), mais aussi feu, souffle, joie, passion, parole, présence. Il suffit qu'il laisse courir sa plume pour que les symboles jaillissent, surabondantes figures de cette puissance qui déborde en lui.

Moins de murmures dans la forêt à la Saint Jean d'été, Il est un moins nombreux ramage en Damas quand au récit des eaux qui descendent des monts en tumulte S'unit le soupir de désert et l'agitation au soir des hauts platanes dans l'air ventilé, Que de paroles dans ce jeune cœur comblé de désirs! (249)

En trois versets, non moins de six métaphores de l'inspiration : rumeurs confuses de la nature, motions élémentaires de l'air et des eaux, tout lui parle et le fait parler, comme cet autre "vates " Victor Hugo.

L'inspiration peut prendre toutes les formes et participer à tout ce que l'homme est, comme à tout ce qu'il ressent : naturelle comme "l'haleine de la terre en culture » (234) et surnaturelle, Sagesse de Dieu qui instruit le poète (248), impersonnelle comme une force de la Nature,

Comme [...] une grande forêt/Que l'on ne cesse point d'entendre dès que l'on cesse de parler [...] (256),

et hautement individuelle, mot suprême de la destinée

parole de la grâce qui est adressée à toi seul (275), 
intime, imperceptible tel le bruissement de "la feuille insistante ", «sibylline » $(246,247)$ - l'inspiration donc se rencontre à tous les niveaux de l'expérience et se mêle à tout mouvement de l'être. Il ne s'agit pas pour le poète de fixer sa physionomie, mais plutôt de nous communiquer à en perdre haleine et jusqu'à l'étourdissement sa richesse inouïe. Inlassablement, Claudel multiplie et diversifie les images; nocturne et diurne -

en moi la fusée de la force nocturne (264)

minute de lumière à voir (244),

l'inspiration est en lui et en dehors de lui : « hors de moi » (264) ou au contraire intérieure à son être comme « la dilatation de la houle » (244). Elle le prend comme la mer qui vient le rechercher (236) et elle prend en lui comme s'élève une acclamation :

Le hourra qui prend en toi de toutes parts comme de l'or, comme du feu dans le fourrage! (233)

Spectacle insolite au vingtième siècle, qu'une poésie où l'inspiration est parvenue à un tel degré de violence! Le poète le premier s'étonne de ce qui lui arrive, lui, l'élève de Burdeau, le fonctionnaire de la Troisième République! La métaphore ironique et amusée, à la fois « naturaliste » et poétique, de « quadrupède ailé " pour Pégase (citée plus haut) traduit sa stupéfaction. Mais aussi sa fierté : car Claudel sait qu'il a bouclé la boucle et qu'en lui se complète le cycle de la grande, la vraie poésie. D'où ces effets de juxtaposition : les manifestations les plus anciennes de l'inspiration, la trépidation de la Sibylle (221), ou

l'ivresse comme celle du vin rouge (231)

rejoignent les plus modernes, telle la pulsation des

frénétiques villes haletantes et tricotantes (267) 


\section{Marie-Joséphine Whitaker}

ou encore l'expérience du chrétien du vingtième siècle qui sent battre en lui la vie de la Trinité :

Entends, comme une vie qui souffre division, le battement de notre triple cœur. (279)

Le dessein de Claudel dans les Cinq Grandes Odes n'est-il pas d'esquisser quelque chose comme une poétique de l'inspiration universelle? Lu sous un certain jour, le poème peut apparaître comme sa métaphore toujours renouvelée : vie de l'universelle beauté grecque dans la première Ode, et dans les suivantes, tour à tour, vent créateur balayant le cosmos de la préhistoire, parole et prophétie bibliques, voix tragique, existentielle de la destinée et enfin vie de la grâce dans l'Église moderne, le souffle se manifeste dans toutes les grandes époques inspirées connues à l'histoire culturelle depuis l'Antiquité jusqu'aux temps modernes, en une recension qui serait complète sans l'omission flagrante du Romantisme que Claudel saute exprès sans doute, injuste envers lui à l'époque ${ }^{3}$ et. se refusant à reconnaître la dette qu'il lui porte.

Son but, en affirmant l'ubiquité, l'universalité et l'unité de l'inspiration est double : rendre hommage à l'Esprit, aussi jeune aujourd'hui qu'il y a vingt-cinq siècles,

l'esprit [...] (qui) n'a jamais à aucun moment cessé de souffler sur l'Humanité (Pr. 523)

et du même coup flétrir le scepticisme d'une ère débilitée et profane, qui peut nier, avec Valéry (Pr. 53) que l'inspiration existe (peut-être par envie?)

Le domaine sacré de l'inspiration, scandaleux pour les déshérités de la grâce, (n’est) blasphémé que par ces gens qui n'en ont jamais franchi le seuil. (Pr. 545)

Et finalement, en une démarche caractéristique de toute poétique au vingtième siècle, Claudel cherche par là à renouer avec la 


\section{La Poétique des Cinq Grandes Odes}

modernité, à présent reliée à la grande tradition; sans nostalgie passéiste, mais sans non plus dévaloriser la poésie. Dans la dernière Ode, le merveilleux se répand silencieusement, sans ostentation mais de manière d'autant plus efficace dans le monde moderne quotidien, «sans aucun son (comme) une rivière d'huile » (280): ainsi s'effacerait la fausse distinction des esthètes, entre l'art et la réalité, le beau et la vie. On ne s'étonne pas outre mesure de voir Claudel rêver, à l'époque des Odes, d'« une grande poésie moderne à la Balzac » (v. C. XII, 172). Mais Claudel n'aurait pu écrire ceci :

\section{[...] les prospectus, les catalogues, les affiches qui chantent tout. haut : Voilà la poésie ce matin [...]}

ni suivre ces autres admirateurs du quotidien, Chesterton et Coventry Patmore (par lui traduits), sur la voie où le poétique s'enlise dans la banalité et le miraculeux se dénature dans l'ordinaire. Car le poète, plus proche des sources de la grande poésie que ne l'est la plupart de ses contemporains, lui est trop fidèle pour oublier qu'elle reste un $\operatorname{art}^{4}$ mystérieux et sacré.

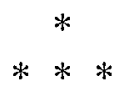

L'inspiration en plus d'un point contredit l'art; une poésie qui coule de source n'a que faire de doctrines; que peut être la poétique d'une ouvre vraiment inspirée? Elle ne sera pas à coup sûr un système de règles ou de formes fixes. À supposer même que le génie de Claudel s'y prêtât, Pierre Brunel nous rappelle à juste titre (à propos d'un autre chef-d'œuvre claudélien) que "l'évolution littéraire » s'y oppose :

Il n'existe plus, an XXe siècle, de " règles " pour la composition d'un chef-d'œuvre : le besoin d'une nouveauté riche, non gratuite, leur a succédé. (Lettres Modernes, Paris $1965,71$. 
On a pu voir à l'origine des Odes, non pas une poétique, mais une " apoétique ${ }^{5}$ Et on peut penser en effet que Claudel écarte d'avance toute notion de composition raisonnée :

Est ce que l'on bêche la mer? (235)

demande-t-il insolemment; et comme il fait sonner haut son défi de poète libre, ayant le droit de procéder "sans art " à l'instar « l'aigle » (224). L'" art ", à l'époque des Odes, prend presque toujours un sens péjoratif sous sa plume :

Ce qu'on appelle art est pour moi moins que rien.

L'art, éternel ennemi des artistes.

L'art d'abord, ou le moyen d'abord ${ }^{6}$ !

Qu'on n'attende pas de son Art poétique qu'il traite de l'art! Dans la première Ode, qui s'annonce comme une «Ars poetica ", une seule Muse s'avère à proprement parler praticienne de l'Art :

La vôtre, avec son cal dans le repli de la main!

Voici l'une avec son ciseau, et cette autre qui broie ses couleurs, et l'autre, comme elle est attachée à ses claviers par tous ses membres! (226)

A-t-on bien compris la plaisanterie? Cette Muse non existante, qui ne figure dans aucune mythologie (pas plus que sur le sarcophage dont s'inspire la première Ode) est inventée par Claudel pour se moquer - dans l'ordre - du « cisèlement " parnassien, de la " peinture verbale »; un troisième trait se voit décoché à l'adresse des « instrumentistes ». Aucune Piéride n'a inspiré René Ghil ${ }^{7}$, et il n'y a pas de Muse de la peinture, ni de la sculpture, encore moins des pianistes (ces derniers simples exécutants). Sont donc épinglés ici finalement les « petits symbolistes » férus de "musique », en un mot tous les techniciens qui se prennent pour des poètes. Les avatars de la Muse qui n'est pas illustrent ce que l'art ne doit pas être. 


\section{La Poétique des Cinq Grandes Odes}

Quelle malédiction que l'art ${ }^{8}$ !

écrit encore l'auteur : mais ne concluons pas trop vite à l'anarchie. Pascal, lui aussi s'est moqué d'une certaine éloquence. Ce que Claudel représente ici en symboles, Baudelaire, l'artiste suprême, l'avait dit en toutes lettres dans «l'Art_romantique ", mettant les poètes en garde contre « le musicien ou le barbouilleur infatigable qui a fait un pacte satanique avec son instrument (O.C. Pléi. 1961, 404) Pacte que Claudel n'a jamais voulu conclure : ne notet-il pas précisément chez lui-même «l'absence d'une subordination amoureuse de l'artiste à son instrument ", soumission qu'il n'a même « pas su feindre »? (C.I. 104). Comment l'aurait-il pu? C'eût été la mort de son lyrisme tout fait de sublimes accents personnels et de présence humaine. Des instincts sûrs guident Claudel dans ses jugements; les exigences du lyrisme, après celles de l'inspiration, règlent sa démarche. Querelle du lyrisme inspiré que celle de l'auteur des Odes, et non pas simple révolte?

Une conviction profonde du poète, c'est que le génie dit " n'importe comment » des vérités essentielles - ainsi travaillaient à ses yeux «Shakespeare, ou Dostoïevsky ou Rubens » (C XII, 204) alors que le technicien avec effort et élégance, ne produit que de l'art. C'est pourquoi « dans la grande poésie lyrique [...] le moyen doit se faire oublier » (Pr. 41) Le moyen. et l'auteur au sens pascalien : c'est l'artiste avec son « caquet prosodique » et ses techniques d'autant plus tapageuses qu'il a moins à dire qu'il faut réduire au silence. Aucune poétique ne supprime l'artiste et l'art aussi impitoyablement que celle des Odes :

Que je ne sache point ce que je dis!

que je sois anéanti dans mon mouvement! (227)

que je sois entre les hommes comme une personne sans visage $[\ldots](283)$

Mais dans la poésie claudélienne, cette suppression libère l'homme. La théorie surréaliste, qui elle aussi veut rompre avec l'art pour 
l'art, mais transforme l'écrivain en médium, ne conduit pas aux mêmes résultats.

$$
\begin{aligned}
& * \\
& * * *
\end{aligned}
$$

Henri Guillemin nous présente Claudel sans plus comme « un artiste ${ }^{10}$, mais l'auteur des Odes accepte avec réticence même le titre de poète et uniquement « à condition d'en écarter toute idée de métier, de mécanique et d'artifice »(Pr. 1475, Notes). L'artiste et le poète, l'art et la poésie s'opposent manifestement dans l'esprit de Claudel en une antinomie inéluctable que nourrissent en même temps l'aspiration à une création vivante et l'aversion pour le « littératurisme » symboliste. Ces positions, au centre de la poétique des Odes, rejaillissent sur l'écriture : écrire de la poésie et parodier l'art, tel est plus d'une fois le but qu'il se propose. Il semble qu'ainsi s'expliquent les gaucheries volontaires qui viennent s'ajouter aux disparités d'une poésie inspirée. Claudel comprend trop bien les naïvetés voulues de Jammes pour que l'on puisse penser autre chose des siennes :

Les gaucheries sont certainement volontaires chez un homme aussi habile que Jammes et ont pour objet l'établissement des valeurs et la rupture constante qui réveille l'attention (C. XII 202).

La gaffe artistique, destinée à mettre hors d'atteinte de l'art des réalités sublimes : est-ce si mal trouvé, le procédé qui consiste à lancer le vers de toutes ses forces contre terre pour qu'il rebondisse vers le ciel, à descendre plus bas que l'art pour monter plus haut que les étoiles? Le vers claudélien chante ou boite, c'est le balbutiement ou le violon, l'impair rugueux ou l'indicible beauté. Ce qui est entièrement éliminé, c'est la zone intermédiaire de l'art, où se déploie la simple habileté de l'artiste. D'après l'économie particulière des Odes, il faut avoir écrit l'abominable vers 


\section{La Poétique des Cinq Grandes Odes}

suivant - le pire des Odes, et l'un des plus mauvais qui soient jamais sortis de la plume d'un poète :

La Force est au midi....

Pareille à ce sûr Thébain sur qui l'œeil du Chef tomba quand il répondit au messager qui racontait Capanée :

"Qui choisirons-nous contre ce contempteur des dieux?»(286)

pour avoir le droit d'écrire ceci :
Ô phrase de l'alto juste et fort, ô soupir de la forêt Hercynienne, ô trompettes sur l'Adriatique! (229)

Il faut avoir employé le mot le plus familier de tous, «truc » (281) pour pouvoir dire ensuite :

La nuit, un rayon d'or sur La Voile, et dans la splendeur du matin, un moment, Une face radieuse [...] (223)

Le prosaïque coudoie le sublime dans les Cinq Odes; mais ce n'est pas la prose - le poète voudrait " marier ses ressources à celles du vers » (Pr. 43) - qui est l'ennemie de la poésie, c'est la littérature :

\section{aboli bibelot d'inanité sonore.}

Puisque le néant s'exprime si bien, ayons l'air d'écrire mal quand nous sommes élus du verbe. Avant d'énoncer une vérité de valeur, ou, mieux, au moment même où elle s'inscrit dans les mots, il faut trouer le tissu artificiel et livresque, démanteler le langage. Faire la nique à la littérature au moment même où l'on nomme la source sacrée - voilà bien l'esprit des Odes. Est-ce une simple coïncidence si Claudel, quand il prononce pour la première fois le mot " inspiré(es) » dans le poème, fait traîner la phrase, lui ôte tout rythme et, lui donne une tournure primaire? 
J'ai dit les Nymphes nourricières; celles qui ne parlent point et qui ne se font pas voir; j'ai dit les Muses respiratrices, et maintenant je dirai les Muses inspirées (225).

L'emploi des moyens trop simples, parodie de ceux, trop compliqués, trop obliques de symbolistes comme Mallarmé, est une autre défense contre l'idolâtrie de l'art. Tout en la désapprouvant, Gabriel Marcel décrit admirablement la manière claudélienne :

On sait l'usage que font les photographes du verbe surexposé. Mais c'est bien ici d'une surexposition qu'il s'agit. L'esprit se cabre contre l'énormité des moyens auxquels il est fait appel pour lui imposer une certaine évidence ${ }^{11}$.

Imposer l'évidence méconnue : n'est-ce pas justement ce que veut Claudel? Le moyen trop gros, rejeté par le lecteur, «se fait oublier », et le force à regarder la chose, à entendre le sens; c'est ainsi justement que se « réveille l'attention ». Le vrai but est d'assurer l'efficacité de la parole : la plaisanterie stylistique est proportionnée chez lui à la gravité intrinsèque d'une poésie solennelle, comme l'est toujours le lyrisme. C'est le sacrifice délibéré de la manière à la matière. L'attitude mallarméenne et valéryenne, qui consiste, au grand scandale de Claudel, à privilégier les mots aux dépens du sens, à leur laisser « l'initiative » (M.I. 79) lui paraît une trahison, pire, un signe de mépris.

Il ne prenait pas les êtres et les choses au sérieux, dira Claudel de Valéry, disciple de Mallarmé (ibid.) Admettons que Claudel parfois écrit " mal » ${ }^{12}$ dans les Odes, mais cela pour sauver le sérieux de la parole. Son vers s'avance, tel un puissant engin, son conducteur déterminé à dissiper tout malentendu, à rabattre la poussière qu'il soulève sur sa route. «Poussière aux yeux », c'est précisément ainsi qu'il arrive à Claudel de désigner un lyrisme non châtié ${ }^{13}$. La littérature ne connaît peut-être pas d'autre exemple d'une telle alliance : lyrisme et réalisme, abandon inconditionnel à l'inspiration et opposition absolue au «sortilège » (265) incanta- 
toire, le plus grand lyrique du vingtième siècle ${ }^{14}$ en quelque sorte son propre Annoncier. Car il n'est pas interdit de penser que la sévérité vigilante de l'esprit claudélien, présente dès le début, s'est incarnée quarante ans plus tard, à l'époque du Soulier de satin, dans cette joyeuse figure de la démystification ou "Verfremdung ». Le réflexe brechtien n'est pas étranger à l'écriture des Odes : réflexe d'une œuvre éprise d'authenticité comme l'est celle du dramaturge allemand, et comme l'était la pensée de tout le siècle révolu. Une certaine tendance à se démystifier soi-même est chez Claudel preuve et acte de foi. Suivons-le une fois de plus dans des développements plus tardifs, là notamment où Animus, sûr de la beauté d'Anima, nous invite à la voir chez elle, ceinte d'un tablier, et livrée « à ses occupations domestiques ». « Vulgaire et par là à mon goût plus émouvante »- ajoute le commentateur - que les "fées préraphaélites » et " princesses de légende » qui peuplaient l'art fin de siècle de «(s)a génération " (Pr. 190-191, 1934) Le ton que le poète et le critique adopte par moments avec nous fait songer au comportement d'une personne sincère, qui, voulant être sûre d'être aimée pour elle-même, se montrerait à nous dans ses mauvais jours et parfois, pour nous mettre à l'épreuve, nous parlerait d'un ton bourru.

$$
\begin{gathered}
* \\
* * *
\end{gathered}
$$

Verve, sévérité et exigence : on retrouve ces attitudes lorsque Claudel aborde les questions de versification ou de prosodie - qu'il jette le ridicule sur le symbolisme d'obédience parnassienne, ou exprime ses opinions sur l'alexandrin Le poème authentique devra s'affirmer d'emblée autre que ces objets trop bien faits dans "l'échoppe "

\section{Du Parnasse Contemporain où l'on fabrique}

Ces sonnets qui partent tout seuls comme des tabatières à musique (O.P. 599). 
(On songera au «Vase brisé » de Sully Prudhomme « ce grand poète! "ibid. 602). Cette intolérance s'enracine dans l'esprit du $\mathrm{XX}^{\mathrm{e}}$ siècle, époque où le poème vivant prend conscience de ce qu'il peut et ne peut plus être en contemplant les produits de la fabrication dus à la machine. Tant pis (ou tant mieux) pour la poésie si beaucoup d'aspects qui avant paraissaient naturels alors se révèlent artificiels, qui auparavant pouvaient se faire passer pour vivants, à présent se font connaître comme mécaniques. La chose fabriquée et son excellence factice, la machine et sa précision d'automate auront porté atteinte pour toute une génération à l'idéal de perfection en art. Chez Claudel, comme chez Bergson, comme plus tard chez Thibaudet, la création s'oppose à la fabrication, la conception vivante à la production en série, le poème à l'artefact - ou, pour emprunter un terme moins spécialisé au vocabulaire comique de Claudel lui-même - à la «souris mécanique » :

Et voilà le mystère, on pourrait dire le scandale de la poésie. Tous ces braves gens qui se sont donné tant de mal, qui se sont tendus des quatre membres en gémissant sur le chevalet de la prosodie, qui ont tant de mérite, tant de bonne volonté, tant d'acquis, tant d'honnête respect pour les règles, tant de loyale sueur sur leur front, et le résultat est une petite souris mécanique qui ne parvient pas à fonctionner. (Pr. 554)

La chose fabriquée et son excellence factice, la machine et sa précision d'automate auront porté atteinte pour toute une génération à l'idéal de perfection en art. Comment ne pas prendre le parti, dès lors, ayant répudié la « perfection stupide ». (Pr. 385)

[...] en toutes choses d'inventer (seulement) l'approximation la plus exquise, toute beauté dans son insuffisance? (O.P. 242) 


\section{La Poétique des Cinq Grandes Odes}

Claudel n'est pas seul en ceci : durant les mêmes années, Péguy devait formuler nettement sa méfiance à l'égard des « parfaits appareils de mécanismes » et des "impeccables serrureries " :

Les grandes choses du monde n'ont pas été des choses bien faites : quand c'est si bien fait que ça, ça ne réussit jamais... il manque justement de ne manquer de rien, ce on ne sait quoi [...] Les vers les plus beaux ne sont pas ceux dont on s'est occupé tout le temps ${ }^{15}$.

Pour des raisons légèrement différentes, un Suarès, un Gide expriment, des opinions analogues :

La perfection n'est pas la poésie ${ }^{16}$.

Le souci de la perfection aujourd'hui semble ne faire que gêner tous ceux qu'il ne fait pas sourire ${ }^{17}$.

La grande tradition poétique rencontre ici les instincts les plus modernes. L'inachèvement sublime conseillé par les anciens traités de rhétorique trouve sa confirmation, dans une poétique du vingtième siècle, dans l'opposition à la machine. L'Ode à la fois antique et moderne peut s'autoriser pour sa « savante négligence » (C. XII, 172) tout aussi bien du « dormitat Homerus » d'Horace que de l'hostilité moderne à l'égard des «froide(s) horlogerie(s)» (Pr. 47).Ce qui se définit à l'époque et s'exemplifie d'avance de manière éclatante dans les Odes, c'est un type de perfection vivante, aussi éloigné que possible du tout fait, du « tout d'une pièce »(Pr. 750). Type irrégulier :

J'écris d'inspiration, j'aime l'irrégularité pour elle-même

(Claudel, interrogé sur son orthographe et ponctuation, C XII, 197, en 1912.)

Asymétrie, imprévisibilité, marques d'un univers traversé par le souffle, où se trouverait réduite au minimum la part de l'automatisme, avec ce qu'il comporte de régularité ou de répétition. 
Polymnie, dans "Les Muses " il est vrai, répète; mais en quels termes, et de quelle manière? À la parole du Créateur : «Qu'elle soit! », elle répond "Qu'elle est » non sans changer, observons-le, le mode du verbe. Et si le poète ajoute quelques vers plus bas -

Toute parole une répétition (230),

ce n'est pas pour nous faire entendre autre chose qu'un écho vivant de Dieu. L'assentiment de « la Muse du poète " (229) ne doit en aucun cas être lu comme mécanique. Polymnie ne répète pas tel un perroquet, encore moins comme une "boite vocale " réglée d'avance. Le même poème (à la page 228), qui recrute des « acteurs " pour représenter le drame du monde, semble d'ailleurs investir "répétition " d'un sens théâtral. Le vocabulaire actif du passage la «troupe ", les « jeux "- chasse loin de l'esprit du lecteur toute vision de vie figée dans la routine. Le vrai sentiment de l'auteur des Odes, c'est que :

Tout mouvement régulier et mécanique est quelque chose d'assommant (Pr. 791).

- et il faut prendre le dernier terme dans son sens premier de «tuant la vie ». La fabrication, la tabatière à musique se répètent, la création, jamais!; dans le domaine du rythme moins qu'ailleurs. À l'intérieur de cette même première Ode où résonne le mot « répétition », la «mesure interrompue » par Erato rompt de façon spectaculaire (231) le schéma établi par l'auteur lui-même. « Dieu seul est assez fort pour exulter dans la monotonie » note Claudel non sans humour dans son Journal (I. 90, en 1909). Si, vingt ans après les Odes on l'entend jouer avec l'idée de la « monotonie » comme « un des besoins essentiels de l'âme humaine » $(\mathrm{Pr} ., 792)$, ce serait dans l'esprit de quelqu'un qui voudrait goûter au moins une fois dans sa vie au plat qu'il avait toujours refusé.

Quelle eût été la réaction de notre poète devant un titre comme : Les Cinq Grandes Odes de Claudel, ou la poésie de la répéti- 


\section{La Poétique des Cinq Grandes Odes}

tion ${ }^{18}$ Peut-être aurait-il attiré l'attention du critique sur cette note du Journal :

La répétition dans la nature a parfois quelque chose de passionné, comme quelqu'un qui veut se faire comprendre? (J. I. 90)

Nous nous substituons à lui pour signaler le propos, croyant reconnaître dans ces accents passionnés ceux du Créateur, ce « quelqu'un » qui s'adresse aux hommes par l'intermédiaire de sa création, c'est-à-dire de la Nature. Pour le poète religieux, écrit encore Claudel,

La Nature est comme un homme qui dit et redit toujours la même chose, comme si ce quelque chose était d'une importance considérable (Pr. 63, en 1927).

Ajoutons par ailleurs que la répétition verbale elle-même, si admirablement analysée par Gérald Antoine, s'avère porteuse de variété dans la poétique des Odes, puisque la reprise, jamais identique, met en relief l'inépuisable richesse de l'expression et a pour fonction d'épaissir, d'achever la pensée (non pas de l'asséner). Comme la Nature, la vie dont Claudel connaît si bien les modalités - loin de se dépenser en de stériles redites - dynamique, tenace, vigoureuse, revient à la charge. L'écriture poétique suivra son exemple, surtout si celui qui tient la plume aura écouté Pindare. «Forge ton langage sur l'enclume de la vérité », celui-ci conseillet-il au poète ${ }^{19}$.

S'il est un tempo que les Odes veulent capter, c'est cette vibration indéfectible que Claudel ressent dans l'univers dès 1904; mais il s'agit dans ce cas d'un rythme créateur, "voulant », qui " compose " (O.P. 163); et on notera que le premier adjectif au féminin, «voulante », revient sous la plume de l'auteur deux ans avant sa mort (Pr. 1392). En effet, que ce soit en 1904, en 1953, ou encore en 1927, il est question de bien autre chose que de ce « battement de tournebroche ", notamment celui de l'inexorable 
"métronome " qui règle l'alexandrin (ibid. 30) tel que le perçoit Claude ${ }^{20}$ Le vers traditionnel, incapable selon lui d'épouser les rythmes du monde, reste étranger aux êtres, indiscret comme cette voix de la maîtresse de piano « qui ne cesse de hurler à notre coude : Un-deux-trois-quatre-cinq-six! » (ibid.). Combien différent le «nombre " de la poésie authentique, en définitive « introuvable» (ibid. 61). Mais en dehors de la prosodie, nous nous trouvons ici devant un problème de civilisation. Qui nous dira jusqu'à quel point le rythme continu, brutal, régulier de la machine que tout moderne a dans l'oreille, est responsable du triomphe du vers libre où notre esprit fatigué cherche le délassement?

Quel soulagement! Comme l'esprit jouit de cet affranchissement de l'oreille! (Pr. 29)

L'irrégularité absolue du vers des Odes, où la recherche la plus systématique n'a pu découvrir d'uniformité et qui continue à défier toute analyse prosodique ${ }^{21}$ - représente le " non " de la vie à l'automatisme. Que l'on relise les «Réflexions sur le vers français »la hantise du mécanique y reparaît à tout moment. Crainte non sans fondement dans le cas de Claudel, d'autant que le vers régulier chez le poète peut par moments révéler son emprise. Etiemble a raison selon nous, pour injuste qu'il puisse se montrer par ailleurs, de signaler la monotonie, le rythme machinal du vers rimé claudélien. Et il n'est pas sans intérêt de noter que les vues du critique rejoignent celles de Louis Prosper Claudel, le père de Paul, qui apprécie son " genre par alinéas » (CI 114), mais juge les vers de douze pieds de son fils (ibid. 123) «froids, exsangues, tirés par les cheveux » et « pas dans (s)es cordes ${ }^{22} »$.

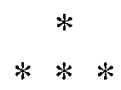

Un dernier écrit critique où s'opposent, dans un autre contexte, artifice et création, nous permet de cerner de plus près encore la 


\section{La Poétique des Cinq Grandes Odes}

conception claudélienne du poème vivant : Francis Jammes a inspiré les lignes que l'on va lire :

À côté de l'artifice [...] de la fabrication, il y a ce langage qui vient de l'âme et qui retourne au cœur ...un mot seulement parfois, une syllabe mais savante à délier le lien mortel [...] une image mais neuve [...] quelque chose jailli de l'inconnu, donné, gratuit, trouvé, dont il sera impossible de dire comment c'est fait (et en effet cela n'a pas été fait autrement que l'enfant par sa mère). ([Pr. 545, en 1917] Nous reviendrons sur ce passage capital)

Texte lourd de sens pour qui se souvient que le poète devait être père trois fois avant la publication des Odes. Dans l'expérience unique de la paternité, la Vie et la Nature l'ont instruit et ont partagé avec lui leurs secrets :

Etreins le texte vivant et ton Dieu invincible dans ce document qui respire, (259)

lisons-nous dans le «Magnificat ». L'identification du créateur avec le père, et du poème avec l'être engendré entraîne des conséquences pour la poétique claudélienne que nous essayerons de dégager dans la dernière partie de cet essai. Dans ces métaphores on sent comme un voile qui se déchire pour séparer à jamais dans l'esprit du poète le vrai du vain (Pr. 805) D'un côté, la fabrication, activité profane, productrice de littérature; de l'autre, la création acte sacré, source d'une existence réelle, participant à la vie divine. Différence inconciliable! D'une part, l'écrit authentique où le poète a pu consigner son être en un acte vital suprême, acte qui engage les forces de l'univers. L'œuvre naît

exigé(e) selon l'ordre de nos générations (O.P. 258),

ayant sa place dans la famille humaine. À côté, en contraste, la. composition fortuite, la fantaisie factice, «individuelle et creuse » 
(Pr. 805) qui flotte, impropre à s'insérer dans le réel. Les symboles de la génération (mot à sens double que Claudel va exploiter), de la procréation et de la naissance, qui jalonnent les Odes, Volker Werner Kapp l'a montré ${ }^{23}$ n'y figurent point pour des raisons simplement autobiographiques : elles ont pour fonction de formuler une certaine conception de l'écriture.

Conception dont il convient de relever les traits organiques. Poursuivant notre investigation, nous observerons avec Claudel que le fabricateur et le technicien ne peuvent s'incarner dans leur œuvre, faite volontairement, consciemment, en obéissance à des règles artificielles, mais en même temps d'après le caprice de l'artiste. « Maîtres de leur matière qui en font à leur gré » ce qu'ils veulent, dira notre auteur en $1922^{24}$, ils nous offrent de l'impersonnel, arbitrairement jeté dans telle ou telle forme - car du point de vue vital, la forme simplement littéraire sera toujours arbitraire - donc quelque chose qui ne ressemble à rien de vivant. Le vrai poète par contre aura le droit de dire

Il naît de moi quelque chose de nouveau avec une étrange ressemblance (258)

Le texte engendré (ou généré) dans la passion ${ }^{25}$, se présente tel l'« opus genitum non factum » (du Credo), ainsi que l'auteur des Cinq Grandes Odes le proclamera hautement, l'écrivant d'abord à Rivière en 1912, ensuite à l'Abbé Douillet en $1922^{26}$. Dans ce cas, cela qui est obscurément formé « dans l'ignorance » (258), alors que « rejoint aux générations de la nature », porte la marque de la nécessité (258). Apparenté à son géniteur, l'être nouveau qui vient au monde n'en reste pas moins indépendant, autre. Et c'est sans doute en hommage au mystère qui accompagne toute parturition, que Claudel, en 1907, qualifie la "nouvelle venue », sa propre fille (ibid.), du curieux nom d' « étrangère »; le terme, tantôt adjectif, tantôt substantif, reparaissant cinq fois dans l'espace d'une vingtaine de vers 


\section{La Poétique des Cinq Grandes Odes}

De moi-même il naît quelque chose d'étranger; un petit homme [...] ordonné pour une fin qui m'est étrangère Ô enfant né sur le sol étranger....! (259)

Qui a suivi le poète dans le déploiement des métaphores relatives à la création ne s'étonnera pas de cette reprise du mot sous toutes ses formes. Il s'agit d'établir, à l'intérieur de la chaîne de vie, la liberté de l'issu(e), poème, enfant, ou parole. Ce sujet amène sous la plume claudélienne de superbes figures - des plus inattendues parfois - justifiées cependant par le contexte. Certaines, et non des moins révélatrices, remontent au tout début de l'œuvre; à La Ville II notamment (1893-7), où l'on entend Avare (l'une des voix du poète dans la pièce) articuler ceci :

En moi, comme la femme qui dans son cour éprouve la commotion de l'enfant mâle, vit

La parole ...et cela, comme un captif,

Avec propriété réclame l'action et la liberté (Th. I, 453)

À leur tour, les Odes feront état de la féminité du poète, la traduisant par un simple cri : « $\hat{O}$ la femme qui est en moi! » (267), mais donnant lieu ailleurs à une comparaison élaborée, qui assimile le créateur fécond à... une nourrice :

Comme la nourrice encombrée de son lait débordant, le poète (l'est) de cétte parole en lui à d'autres adressée (259)

Qu'eût dit Boileau!? La réunion du féminin et du masculin, la superposition des modes de la paternité et de la maternité était cependant indispensable : elle apporte au poète les moyens de formuler une idée qui lui est chère : celle, notamment, de la forme intérieure, propre à la grande poésie. La notion se laisse appréhender en un premier temps au plan du langage critique, énoncée dans ce style par l'auteur des Réflexions en 1927 (Pr. 40) : 
Quand nous aurons fourni au vers en lui-même sa propre subsistance, il n'aura plus besoin d'aller chercher au dehors appui...

Et l'auteur de nous donner l'exemple de ce vers idéal, qui n'obéit à rien d'extérieur, mais réalise cet intime désir " d'action " autonome que lui prêtait Avare :

[...] pour la première fois, dans Francis Jammes, nous sentons la poussée d'un vers qui compose lui-même son nombre et son rythme (M.I. 192)

Francis Jammes : rien de fortuit dans sa réapparition. On ne l'a pas oublié, c'est lui qui suscitait, dans le texte précédemment cité ( $\mathrm{Pr}$. 545, 1917), la figure-clé du poème prenant forme comme l'enfant dans le sein de la mère. Métaphore qui jette une lumière nouvelle sur le sujet : dans la relation du poète avec l'œuvre se voit ainsi inclue la gestation; immensément enrichi, ce rapport s'inscrit dès lors dans les processus de la nature elle-même, et présuppose une lente, secrète communication de substance à substance. Dans une telle perspective, quelle sera la part du travail poétique? On comprend qu'il restera dans une grande mesure caché, soustrait à tout regard et à tout calcul, essentiellement distinct de l'effort délibéré de l'artiste. Telle la mère, ce dernier - dans la configuration que nous propose Claudel - fournit sa « subsistance » (cf. supra) à l'être en lui vivant, tirant le suc nécessaire de ce qu'il est lui-même. Le fabricant ou technicien, ne l'ayant pas porté en lui, travaille trop l'objet de manière purement extérieure, cherche « au dehors » et appelle cela «forme »; le créateur, comme la mère, sait qu'il ne peut agir sur le poème par pression externe : sa « constitution » apportera à « l'enfant » la matière dont il a besoin. Comme la mère aussi, il reconnaît que son œuvre naîtra déjà formée. Transposé en termes concrets : au lieu de torturer le texte, nourrir l'écrit d'aliments et appropriés - tel serait le principe claudélien.

Ces aliments? Contrairement à ce que l'on pourrait croire, s'agissant d'un poète profondément religieux, la liste dressée par 


\section{La Poétique des Cinq Grandes Odes}

Claudel en 1925 à l'intention des « jeunes écrivains » comprend force nourritures terrestres : science, affaires, droit social aussi bien que théologie leur sont recommandés (Pr. 43). D'après le diagnostic de leur aîné, en effet, l'anémie et la débilité guettent une gent littéraire ignorante du monde. Et lorsque deux décennies plus tard, Claudel déclare « prodigieuse » l'ignorance de Mallarmé ${ }^{27}$, il réaffirme par là ses positions, prenant une fois de plus le contre-pied de la Décadence.

"Que les vers ne soient pas votre éternel emploi "; " connaissez la ville »; " que votre Muse en savantes leçons soit fertile ${ }^{28}$. Claudel, nous aurions voulu le montrer, remet au premier plan, les ayant redécouvertes, les conditions naturelles dans lesquelles naît une grande œuvre ( « redécouvertes ", disons-nous, parce qu'obnubilées par l'idolâtrie de l'Art et l'ésotérisme fin de siècle). L'auteur des Odes renoue ainsi avec un bon sens séculaire. Car aucun Art poétique classique, depuis Horace jusqu'à Boileau, ne cesse d'insister sur le rôle formateur de la vie et de l'expérience, ou encore sur la qualité d'être de l'écrivain, qui transparaîtra, quoi qu'il fasse, dans ses écrits. Faut-il rappeler que « Le vers se sent toujours des bassesses du cœur »? Et si Boileau retourne et transpose ici en plus pédestre le propos de Longin; il reste que, dans les deux cas se voit réduite l'importance des questions « d'art ».

Seuls ceux qui ont de hautes et solides pensées (peuvent) faire des discours élevés; c'est aux grands hommes qu'il échappe de dire des choses extraordinaires ${ }^{29}$.

$$
* * *
$$

La Poétique des cinq Grandes Odes - titre adopté par cet essai - ou plus simplement, poétique de l'Ode? Grand connaisseur du genre, André Suarès salue en Claudel l'unique héritier au temps moderne des Grecs, praticiens de cette forme en tant qu' « œuvre d'art » ${ }^{30}$. Les dernières pages de cet essai voudraient jeter un regard sur la spécificité de l'œuvre sortie des mains de notre poète dans la première décennie du $\mathrm{XX}^{\mathrm{e}}$ siècle. Entreprise difficile, mais non 


\section{Marie-Joséphine Whitaker}

impossible, à condition d'écouter Claudel parler « en poète de la poésie »(Pr. 40). Car l'Ode se manifeste en une série de figures dont il n'est pas toujours aisé de saisir le sens, d'autant qu'au lieu de s'expliciter, elles se contentent de nous éblouir. Ainsi de «L'Ode soudaine » qui jaillit sous la plume de l'auteur en une subite « déflagration ", frappant " de tous côtés comme l'éclat de la foudre trifourchue » (223). Dédiée à Mnémnosyne, mère des Muses, elle réussit néanmoins à établir la coïncidence du langage qui l'exprime dans le temps, avec l'incréé, ou, dans les mots du texte, avec « le pouls même de l'Etre ». Une telle dédicace nous invite à suivre l'auteur sur la piste de l'Ode, écrit sacré : caractère de sacralité qui ne se dément point lorsque l'«Ode pure » personnifiée en athlète (229) invite le Panthéon grec à se joindre aux triomphes des hommes. Rapporter ce rite, c'était pour Claudel marquer sa fidélité à un genre qui " entretient dans ses vers commerce avec le Dieux " (Boileau, 31). Et si sa graphie leur ôte la majuscule, - « tous les dieux » se lit la page 229 - rappelons que le poète a toujours préféré les païens religieux à ses contemporains incroyants. Aussi bien, l'Ode que Claudel offre au Dieu chrétien (235), se présentera " nouvelle ", et simultanément, comme ne connaissant point de commencement, inscrite comme elle l'est, hors de toute confession, dans l'esprit religieux universel. Ainsi dès le début s'opère sans heurt la transition du sacré antique au sacré chrétien.

L'intermédiaire désigné aura été, on le sait, le " radieux Pindare » (228), une des « sources » de l'auteur, et son « réconfort littéraire ${ }^{32}$. Il était impératif pour lui, dès «Les Muses », de rendre hommage au chantre de l'énergie divine et de l'exploit, antidote prédestiné aux langueurs symbolistes. Mais en même temps les Pythiques de Pindare, indéniablement un modèle, lui proposaient un patron dynamique annonçant le type d'ode qu'il souhaitait lui-même réaliser. L'ode ou poème-acte « action humaine " créant "le temps nécessaire à sa résolution ", ces métaphores (ibid.) captent mieux que toute autre ce qu'il veut représenter - à savoir, un ensemble à la fois diversifié et concerté, mouvant et unifié ${ }^{33}$. 


\section{La Poétique des Cinq Grandes Odes}

\section{Ô la grande journée des jeux! (228)}

Les « jeux " pindariques lui en fournissaient l'exemple éclatant. Mais l'a-t-on remarqué? Aux jeux au pluriel succède le jeu au singulier, auquel le poète participera avec passion («mon ardente part au jeu » lirons-nous dans la deuxième Ode, 239). Mais ici, (230) il s'agit du Jeu par excellence, disposé " avec beauté » par Dieu, c'està-dire de la Création, "énorme cérémonie ", déclenchée; donc perçue par Claudel comme une solennelle mise en marche de forces qui ne s'épuiseront pas de sitôt. À preuve l'expression enrichie qui orne la page suivante : "l'inexhaustible cérémonie vivante ». Vivante et qui regroupe des vivants, d'une manière qui n'interfère aucunement avec leur liberté. Un vers qui a pu passer inaperçu, rarement commenté, pose les bases de la conception :

\section{L'Or, ou connaissance intérieure que chaque chose} possède d'elle-même (229)

Chacun a un nom qui lui est propre, et il obéit d'instinct lorsqu'interpellé par ce même nom; ainsi en fut-il pour Claudel au moment de sa conversion ( $v$. «Magnificat » 249). À son tour, l'auteur imite Dieu (230), appelant l'un après l'autre les existants de la même façon. C'est dans ces conditions que « toute chose » rentre volontairement « tour à tour » (229) dans l'ensemble, "l'ordre » « impos(é) » (230) n'étant pas ressenti comme impropre ou étranger. Si le poète évite l'écueil, c'est qu'il « nourrit » en lui « rassemblement " et « dissolution» (230), ce qui exclut la forme fixe, au bénéfice de la « danse sacrée » qui laisse latitude aux participants ${ }^{34}$. Découvrir la formation naturelle acceptable aux créatures, qui ne leur ravit point leur identité ni leur autonomie, s'avère être l'une des tâches essentielles dévolues à l'Ode. Dès la deuxième, «L'Esprit et l'Eau », on saisit la démarche : composer un poème sacré, c'est faire passer les êtres d'une zone triste, décolorée, désensibilisée par l'habitude (cette ennemie mortelle de la poésie, Pr. 48) et l'ennui, dans un monde resplendissant de joie ${ }^{35}$, de vigueur, de spontanéité. Ainsi l'action du poète est double : 


\section{Marie-Joséphine Whitaker}

J'apporte à toute chose la délivrance

Par moi

Aucune chose ne reste seule, mais je l'associe à une autre dans mon cœur (238)

Les êtres, «seuls » dans un monde sans Dieu - et dans les « arrangements cruels » (235) d'un art profane, qui ne peut que dénaturer, désunir ou tyranniser, se regroupent dans l'ode pour la jubilation et la célébration, d'après un principe affectif et naturel. Ce n'est que dans une telle configuration que chaque chose peut dire ce qu'elle «veut dire » (231); les structures d'un monde désacralisé étouffent le désir d'être pleinement, qui n'est autre pour Claudel que l'instinct vital, dont on ne réprime pas impunément l'élan. Dès La Ville il posait, en langage imagé bien entendu, le principe d'une révolte contre les barrières qui séparent la créature de Dieu, son vrai maître. L'âme qui brise tout pour le rejoindre : de superbes vers représentent son cas de façon inoubliable :

[...] comme une jument vierge, la bouche embrasée par le sel qu'elle a pris dans la main de son maître Comment... la serrer et la contraindre, la grande chose terrible qui se dresse et qui crie, dans l'étroite écurie de sa volonté personnelle? (Th. I, 480)

L'écriture des Odes épouse plus d'une fois la cause des violents, de ceux qui s'insurgent contre le statisme, l'inertie, le poids de la matière; le poète ne craint point de puiser des exemples dans la Bible (en l'occurrence dans le Psaume CXIII, 6)

Et la montagne dans l'éclair qui la montre et qui l'absorbe tour à tour a l'air de sauter comme un bélier (253)

Une « chose vue » pourrait inspirer le vers qui suit - 


\section{La Poétique des Cinq Grandes Odes}

Comme une barque qui ne tient plus qu'à sa corde, et qui danse furieusement, et qui tape, et qui saque, et qui fonce et qui encense, et qui culbute, le nez à son piquet (264).

- n'était le verbe « encense » qui interprète la fureur et lui confère un sens élevé : cet être déchaîné, parvenu au maximum de sa vitalité, en déployant toute l'énergie dont il est capable, accomplit un acte pieux. «Que ma prière monte vers vous, Seigneur, comme la fumée de l'encens » se lit après tout dans le missel ${ }^{36}$ La liturgie des vivants que captent les Odes assemble les extrêmes. Ce que Claudel entend par louange et prière tient entre le geste de Polymnie qui approuve (Dieu) substantiellement (230) et les réactions sauvages d'êtres qui adorent en se rebellant contre l'absence du Créateur. L'âme, « orante » recueillie, prosternée dans la neige (257) et le « petit tigre » qui « se ramasse » pour le bond, la jeune fille fuyant les siens à l'appel du bien-aimé (264), ces images si diverses ont toutes deux leur place dans le poème claudélien.

Si ces attitudes nous choquent, si cette violence originelle libérée dans les êtres nous semble contredire l'esprit d'une poésie religieuse, c'est que nous n'avons guère compris la manière dont Claudel vit et fait revivre le sacré. Aurions-nous oublié le long combat du poète contre le «fade », son contraire symétrique ${ }^{37}$ ? Ce n'est qu'en réconciliant la religion et l'instinct que Claudel pouvait triompher dans l'Ode pindarique. "Indiscutable, dévorant, impérieux » ainsi que le caractérise Roger Caillois ${ }^{38}$, et allié de tout temps avec le sublime, d'après son illustre interprète, Rudolf Otto ${ }^{39}$, le sacré devait trouver son porte-parole en Claudel, un Claudel privilégié et qui reste un cas isolé. Jammes et le Verlaine de Sagesse ${ }^{40}$ sont simplement des poètes pieux. Seul le poète catholique pouvait faire entendre à nouveau, et ceci avec l'accent de son siècle, une voix trop longtemps tue. On s'étonne qu'Etiemble - le catholicisme qu'il abhorre l'aurait-il aveuglé ? - ait pu qualifier l'auteur des Odes de "marchand de vin de messe "! (loc. cit., 491) Eût-il étudié, comme le fera le lecteur non prévenu, l'imagerie et les éléments du sacré qui animent les poèmes, il aurait 
découvert leur profond ancrage dans la nécessité : vêtements liturgiques, montrance, « azyme » (263) ils sont tous justifiés par le genre plutôt qu'ils ne le désignent.

Tout un réseau symbolique, en effet, soutient, illustre et développe la pensée de l'auteur. Que ce soit l'image de son cœur, lourd comme un encensoir, qui s'élève vers Dieu $(250,41)$; ou le vœu exprimé dans le "Magnificat » de réaliser sa vocation poétique en " consacrant », donc en rendant sacrée la parole (tel le prêtre qui meurt à l'autel, 251); quelle beauté s'attache à cette autre figure sacerdotale, qui reçoit « ordination » au lendemain de Noël, l'isotopie revêtant la terre d'une chasuble blanche propre à la saison! (256). La symbolisation la plus étonnante toutefois sera celle qui réunit le célébrant et le père, Dieu ayant voulu " recevoir " "vie » de l'un et de l'autre, aussi bien dans l'hostie «entre les doigts du prêtre qui consacre » (258) que dans « cette image réelle » de Lui-même qu'est le nouveau-né. Remarquons finalement la manière dont le poète amène les Odes à correspondre avec sa vision du sacré, greffant la première (en sa fin) sur la Genèse; en conférant à la deuxième et à la troisième la forme de prières ${ }^{42}$. Conçue comme un dialogue, la quatrième n'en supplie pas moins Dieu (270-271); dans la « Maison fermée » une fois mise en ordre, à partir de la page 288, le poète, "Officiant » (290) offi$\mathrm{cie}^{44}$, s'adressant aux morts et aux vivants, sans oublier toutefois sa fonction principale.

\footnotetext{
Vous voyez cette terre qui est votre créature innocente...

Délivrez-la par ma bouche de cette louange qu'elle vous doit (261-262).
}

Chant de louange, c'est peut-être par là en fin de compte que l'Ode définit ce qui fut de tout temps sa nature et son rôle. On a vu Claudel défendre farouchement contre sa tentatrice $(265-70)$ ce « devoir " de célébration de Dieu et du monde, cette tâche essentielle qu'il considère sienne. En conclusion, la question peut se poser : ne s'en est-il pas acquitté mieux que ses prédécesseurs? Il est 
fort peu probable que Claudel ait songé à l'auteur des Pythiques en écrivant ici même (267) «L'antique poète parlait de la part des

$\begin{array}{ll}\begin{array}{l}\text { Pindarum quisquis studet } \\ \text { aemulari }\end{array} & \begin{array}{l}\text { Quiconque, Julius, } \\ \text { cherche à rivaliser avec } \\ \text { Pindare } \\ \text { Jule, ceratis ope Daedalea } \\ \text { S'appuie sur les ailes que } \\ \text { l'art de Dédale }\end{array} \\ \begin{array}{l}\text { Nititur pennis vitreo } \\ \text { Jaturus }\end{array} & \begin{array}{l}\text { Joignit à la cire, et } \\ \text { Nomina ponto }\end{array} \\ \text { Dounera à la men nom, un } \\ \text { jouristal. }\end{array}$

dieux sans présence ». Il reste qu'on chercherait en vain chez le grand Thébain cette familiarité avec la parole divine que seule la lecture du Livre de Dieu pouvait apporter au poète chrétien, ou cet amour profond des créatures qui marque chaque page des Odes. Dans la nature même des choses, notre poète devait réaliser autre chose qu'un simple pastiche de Pindare. Le surpasse-t-il? Impossible! déclarait d'avance Horace (Ad Julium Antonium, Ode II Livre IV).

Claudel a donné son nom à une place dans Paris. Et il n'a point sombré dans la mer Égée...

\section{Notes}

Remarque : Ce qui est mis en italiques dans les textes cités, sauf avis contraire, l'est par nous. Toutes les références de pages pour les Cinq Grandes Odes renvoient à l'Euvre poétique (O.P.) Pléiade N.R.F., 1967. En Pléiade également Le Journal de Paul Claudel (J. I, J. II) 1968 et 1969, le Théâtre, abrégé en Th., et l'Euvre en Prose, 1965 (Pr.). 


\section{Marie-Joséphine Whitaker}

\section{Abréviations}

BSPC - Bulletin de la Société Paul Claudel, 13, Rue du Pont-LouisPhilippe, 75004 Paris.

C. I, C. XXII - les Cahiers Paul Claudel, publiés par Gallimard N.R.F à partir de 1959. (N.B. Le Cahier XII contient la Correspondance avec Rivière.)

M.I. - les Mémoires Improvisés, N.R.F., 2001.

1 Correspondance Paul Claudel - Francis Jammes - Gabriel Frizeau, N.R.F 1952, p. 130.

2 Voir notre article « Le Vitalisme de Claudel» dans Les Cinq Grandes Odes, BSPC n $51,3^{\mathrm{e}}$ trimestre 1973.

3 « [...] la haine que j'ai pour les Romantiques " écrit-il à Gide. Correspondance Paul Claudel - André Gide, N.R.F. 1949, p. 169.

4 Si Claudel s'en prend à "l'Art pour l'Art » des esthètes, et à l'acharnement des techniciens, il ne nie aucunement la part de l'art authentique dans la composition d'une cuvre. V. dans Pr. « La Poésie est un art »; « L'Art et la Foi ».

5 « Les Cinq Grandes Odes [...] ne forment qu'un bel accident, écrites qu'elles furent dans l'ivresse, dans le vertige [...] quand un chacun s'inventait une apoétique ", écrit Etiemble. "Paul Claudel et le Vin des Rochers ", Hommage à Claudel, N.R.F., n 33, $1^{\text {er }}$ septembre 1955, p. 484.

6 Correspondance Paul Claudel - André Suarès, N.R.F., 1951, p. 97, ensuite p. 185. (Désormais abrégé en « Corr.Su» dans les Notes) La dernière citation figure dans une Lettre à Rivière de 1912, v. C XII, p. 204.

7 René Ghil (1862-1925), dans son Traité du verbe (six éditions entre 1885-1904) propose une "Théorie de l'instrumentation verbale » qui connut nombre d'adeptes.

8 Corr.Su, p. 100.

9 Rappelons: «L'inconvénient de la révolte, c'est qu'elle ne mène nulle part $» \operatorname{Pr}$. 60. A distinguer de l'insurrection légitime contre ce qui bloque le chemin vers Dieu; v, infra.

10 Claudel et son art d'écrire, N.R.F 1955, p. 13.

11 Regards sur le Théâtre claudélien, Paris, Beauchesne 1964, p. 141.

12 «Mauvais poète, pire écrivain " formule d'Etiemble, loc. cit., p. 483. C. « Claudel écrit mal, dans un français rocailleux et plein de fautes 


\section{La Poétique des Cinq Grandes Odes}

de syntaxe $»$. Le BSPC. $n^{\circ} 187,3^{\text {e }}$ trimestre 2007, p. 32 rapporte cet autre jugement, provenant, il est vrai, d'un ennemi politique du poète (1943). 3) V. La Préface, de la main du poète, au livre du Père L. Barjon. Claudel, P.U.F. 1953 p. 8.

14 « Parmi les Français modernes, Claudel figure le seul poète-né, un poète authentique et profond pour qui les choses sont neuves comme au premier jour "Ernst Curtius, cité par Charles du Bos, Approximations, Fayard 1965, p. 1249.

15 Péguy, Note conjointe sur M. Descartes, (1909-1914), CEuvres en Prose, Pléiade 1961, p. 1365.

16 Corr.Su, p. 128.

17 Gide, Journal I, Pléiade 1955 p. 248.

18 Gérald Antoine, Les Cinq Grandes Odes de Claudel ou la poésie de la répétition, Lettres Modernes, M. J. Minard, 1959.

19 Odes Pythiques, t. II, Les Belles Lettres, 1977, p. 33.

20 On sait qu'un an avant sa mort, Phèdre de Racine le fera revenir sur ses préventions. «Ces vers inouïs [...] il était naturel qu'après les avoir tracés, la plume se rompît [...] dans une main sublime » Pr. 466-467.

21 Y. Scalzatti, «Le Verset Claudélien, une étude du rythme », Lettres Modernes $\mathrm{n}^{\circ} 63$ (6) 1965 p. 18-19 évoque le nombre introuvable; Jacques Lefèbvre, auteur de "Lecture du vers claudélien » ( ${ }^{\circ}$ spécial 20, Société Claudel en Belgique 1975) conclut son article « Rythme et syntaxe dans la poésie claudélienne »Belgique, Humanités chrétiennes p. 28 en ces termes «nous laisserons le dernier mot à la liberté, c'est-à-dire, à la poésie... »

22 Il s'agit de vers parus au Mercure de France en 1911. Voir note O.P. 1198. Titres : «Chant à cinq heures » O.P. 7, et « Dédicace », ibid., p. 964.

23 Voir Volker Werner Kapp Poesie und Eros, die Fünf Grossen Oden von Paul Claudel, Wilhelm Fink Verlag, München, 1972, p. 17, 18, 81, passim.

24 Pour la Lettre à l'Abbé Douillet, de 1922 v. Paul Claudel, Supplément aux O.C. I, L'Age d'Homme p. 324, où reparaît une deuxième fois le mot « ignorance".

25 Des trois enfants venus au monde entre 1905 et 1908, l'enfant de la passion serait sans doute le premier, la fille née hors mariage, loin de son père, en Belgique. Certaines inflexions font penser que le poète ne l'oublie pas alors qu'il célèbre la naissance de Marie. 


\section{Marie-Joséphine Whitaker}

26 C XII 203; Lettre à L'Abbé Douillet, citée supra, même page.

27 Dominique Millet-Gérard, « Regards latéraux sur l'exégèse claudélienne » De précieux extraits des Entretiens avec le Père Blanchet ouvrent l'article, et nous fournissent l'opinion sur Mallarmé. Voir $\mathrm{BSPC}^{\circ} 175,3^{\mathrm{e}}$ trimestre, octobre 2004, p. 25.

28 Boileau, O.C., Pléiade, 1966, Art Poétique, p. 183, 178, 182.

29 Longin, Traité du Sublime traduit par Boileau, même volume, p. 351

30 Corr.Su, lettre de 1907 p. 103.

31 Boileau, Art Poétique, sur l'Ode; op. cit., p. 164.

32 Corr.Su, p. 27, Lettre de Claudel de 1904 (elle précède celle citée à la note 30 ) qui remercie Suarès de lui avoir permis de « faire connaissance avec Pindare ".

33 Le poème-acte quitte pour ainsi dire la page pour passer dans le domaine des arts du temps et de l'espace, la danse et le drame-action Les allusions à la tragédie grecque, (p. 228) ailleurs à la comédie, p. 278, confirment ces vues. En plus d'un endroit de son cuvre Claudel cherche à abolir les distinctions entre les genres.

34 Claudel donnera plus tard le nom de « danse sacrée " au mouvement des idées naissantes à l'intérieur d'une écriture agitée par la passion et en rapport avec l'Infini. Lettre à l'Abbé Douillet, op. cit., p. 325 et 326. En 1934, dans son « Commentaire sur le Psaume XXVIII " (Le Poète et la Bible I, N.R.F. 1998, p. 71-72) il associera cette danse avec les impulsions du Saint-esprit, qui inspire les Psaumes, et ensuite avec l'inspiration en général. À l'époque des Odes, en 1906, le poète parlera à Gide (Corr. p. 64) de « l'agitation sacrée de la créature, toujours en état de création " dans " un monde où tout est mouvement ». La même année il écrira à Suarès (Corr. p. 87) que " les choses bougent ineffablement devant l'éternité ".

35 «La joie détruit l'habitude », lisons-nous J. I. p. 502.

36 Mots empruntés au paroissien de l'époque (Offertoire, aux Messes solennelles).

37 Voir Pr.p. 113-121, « Le Goût du fade » et l'essai suivant.

38 L'Homme et le Sacré, N.R.F. 1950, p. 3.

39 Rudolf Otto voit dans le sublime « le moyen le plus efficace pour représenter le sacré ». Voir Das Heilige (1917), chapitre IX, section 3.

40 Il est significatif qu'en 1951 Claudel exprime des réserves sur ce recueil précédemment défendu en 1935 (M.I. 191).

41 On retrouve l'image dans J. I, p. 34. 


\section{La Poétique des Cinq Grandes Odes}

42 Nous avons cherché à le démontrer en détail pour « l'Esprit et l'eau » dans Paul Claudel : Les Odes, édité par Sergio Villani, Albion Press 1994. Il serait encore plus facile de le prouver pour le « Magnificat».

43 L'image récurrente du prêtre, on ne peut trop insister là-dessus, exigée par le caractère sacré de l'Ode, est parfaitement intégrée aux formes poétiques; elle a peu à voir avec la nostalgie du sacerdoce chez Claudel, ainsi qu'on l'a trop dit, réduisant ainsi la. portée des textes. 